\title{
EVOLUCIÓN UROLÓGICA DE PACIENTES CON AGENESIA DE SACRO: 20 AÑOS DE SEGUIMIENTO.
}

\author{
José María Abascal Junquera, Joan Conejero Sugrañes, Raul Martos Calvo, Ana Celma
}

Doménech, Carlos Salvador Lacambra y Pascual Zamora Escamez.

Unidad de Urodinámica. Servicio de Urología Hospital Vall d’Hebron. Barcelona. España.

\begin{abstract}
Resumen.- OBJETIVO: La agenesia sacra total ó parcial es una malformación infrecuente que consiste en la ausencia de una ó más vértebras sacras. Forma parte del síndrome de regresión caudal y se puede asociar a otras anomalías congénitas (síndrome de Currarino). No tiene una etiología establecida pero se asocia a madres diabéticas insulinodependientes (1\%). El objetivo de este estudio ha sido analizar retrospectivamente la evolución urológica de los pacientes con agenesia sacra de nuestra serie.
\end{abstract}

MÉTODOS: Análisis retrospectivo de 14 pacientes entre 1975 y 2005. Se valoró el motivo de consulta, situación urológica y neurológica de entrada, tiempo de seguimiento, evolución de la continencia, complicaciones urológicas, ingresos hospitalarios y número de visitas ambulatorias.

RESULTADOS: Ningún paciente tenía antecedentes de madre diabética. La distribución por sexos fue similar. Todos los casos fueron de agenesia total. La edad media en la primera visita fue de 13,2 años y el principal motivo de consulta fue la incontinencia urinaria. El $60 \%$ de pacientes presentaban mielomeningocele asociado.

José María Abascal Junquera

Servicio de Urología Hospital Vall d'Hebron Passeig Vall d’Hebron 1 19-129 8035 Barcelona. (España) josuvargas@hotmail.com

Trabajo recibido: 31 de marzo 2006
El $70 \%$ tenían el tracto urinario superior normal al inicio del seguimiento; 10 pacientes presentaban algún grado de incontinencia (70\%). El tiempo medio de seguimiento ha sido de 19,7 años (7-30): el 50\% de los pacientes mantienen el tracto urinario superior normal. El $65 \%$ de los pacientes se mantienen continentes. Todos han presentado infección urinaria sintomática. El motivo urológico principal de ingreso hospitalario ha sido la cirugía programada (7). El número medio de visitas anuales por paciente ha sido de 1,9.

CONCLUSIONES: La agenesia de sacro es una malformación congénita infrecuente que requiere un diagnóstico precoz para evitar complicaciones urológicas a medio plazo. Estos pacientes son subsidiarios de un control urológico de por vida. El principal motivo de consulta es la incontinencia urinaria por vejiga neurógena que puede ser tratada satisfactoriamente en la mayoría de los casos.

Palabras clave: Agenesia de sacro. Vejiga neurógena.

Summary.- OBJECTIVES: Complete or partial sacral agenesis is a rare malformation consisting in the absence of one or more sacral vertebrae. It is part of a caudal regression syndrome and it may be associated with other congenital anomalies (Currarino Syndrome). It does not have an established etiology but is associated with insulin-dependent diabetes mellitus in the mother (1\%). The objective of this is study was to retrospectively analyze the urological outcome of patients with sacral agenesis in our case series.

METHODS: Retrospective analysis of 14 patients between 1975 and 2005. We evaluated reason for consultation, urological status, continence outcome, urological complications, hospital admissions and number of office visits. 
RESULTS: No patient had history of diabetic mother. The number of male/female patients were similar. Mean age at first visit was 13.2 years and main reason for consultation was urinary incontinence. $60 \%$ of the patients presented associated myelomeningocele. $70 \%$ had a normal upper urinary tract at the beginning of follow-up; 10 patients presented some degree of incontinence (70\%). Mean follow-up was 19.7 years (7-30): $50 \%$ of the patients keep a normal upper urinary tract. All of them have presented symptomatic urinary tract infection. The main urological reason for hospital admission was programmed surgery (7). The mean number of visits per year was 1.9.

CONCLUSIONS: Sacral agenesis is a rare congenital malformation requiring an early diagnosis to avoid mid-term urological complications. These patients need life-long urological follow-up. The mean reason for consultation is urinary incontinence secondary to neurogenic bladder which may be satisfactorily treated in most cases.

Keywords: Sacral agenesis. Neurogenic bladder.

\section{INTRODUCCIÓN}

La agenesia sacra total ó parcial es una malformación infrecuente que consiste en la ausencia de una ó más vértebras sacras. Se ha clasificado en cuatro tipos: los tipos 1 y 2 serían casos de agenesia parcial, mientras que los 3 y 4 (ausencia también de alguna vértebra lumbar), serían pacientes con agenesia total. Su incidencia se estima entre un 0.09 y un $0.43 \%$ de la población general. Forma parte del síndrome de regresión caudal y se puede asociar a otras anomalías congénitas como malformaciones anorrectales y urogenitales; ésta tríada es conocida como síndrome de Currarino, y se considera de herencia autosómica dominante $(1,8)$.

La agenesia de sacro no tiene una etiología establecida pero es posible que los factores teratógenos desempeñen un papel importante, dado que las madres diabéticas insulinodependientes tienen una probabilidad del $1 \%$ de tener descendencia con esta anomalía. A la inversa, un $16 \%$ de los niños con agenesia sacra tienen una madre con diabetes insulinodependiente (1-3).

Su detección precoz es esencial por su asociación a vejiga neurógena. El retraso diagnóstico aumenta el riesgo de lesión renal, infecciones urinarias de repetición e incontinencia. Se ha sugerido que el deterioro neurológico progresivo que presentan estos pacientes es debido al crecimiento inapropiado de las raíces nerviosas sacras, anormalmente situadas. Dado que estos niños presentan una sensibilidad normal y un grado mínimo ó nulo de deformación ortopédica en las extremidades inferiores, la lesión subyacente pasa a menudo inadvertida; el $20 \%$ de casos son diagnosticados entre los 3 y 4 años de edad cuando los progenitores comienzan a preocuparse por el fracaso del entrenamiento vesical e intestinal del niño (1-7).

Clínicamente estos pacientes pueden debutar con un estreñimiento crónico. Los síntomas urológicos principales serían la incontinencia urinaria diurna y nocturna, infecciones urinarias de repetición y las dificultades en el vaciado miccional. La exploración física puede demostrar un aplanamiento de las nalgas con un pliegue interglúteo bajo y corto ó incluso inexistente; en ocasiones puede palparse el defecto sacro directamente. La exploración neurológica puede ser estrictamente normal (5-7).

Cualquier defecto del sacro que afecte a más de una vértebra puede causar una disfunción vesical. Sin embargo, si la lesión engloba la zona S2-S4 tiene más probabilidades de ocasionar una vejiga neurógena. La cantidad de vértebras afectadas aparentemente no se correlaciona con el tipo de lesión de la motoneurona presente (1).

El diagnóstico puede confirmarse mediante una radiografía simple de pelvis anteroposterior (Figura 1) ó mejor lateral, dado que esta área a menudo está enmascarada por el aire intestinal ó heces. Algunos autores preconizan el uso de la resonancia magnética nuclear para valorar mejor la médula espinal, observándose casi siempre una interrupción brusca del cono medular a nivel de la vértebra T12 (Figura 2) (2-4).

Los hallazgos urodinámicos demuestran lesión de motoneurona superior en un 35\% de los casos (hiperreflexia del detrusor, hiperactividad de los reflejos sacros, ausencia de control voluntario del esfínter, disinergia vesico-esfinteriana); en un $40 \%$ habría afectación de motoneurona inferior (arreflexia del detrusor, disminución ó abolición de los reflejos sacros). En un $25 \%$ de casos no se observarían signos de denervación al diagnóstico $(1,2)$.

El objetivo de este estudio ha sido la revisión retrospectiva de la evolución urológica de pacientes con agenesia de sacro de nuestra serie.

\section{MATERIAL Y MÉTODOS}

Se han revisado retrospectivamente 14 pacientes controlados en nuestro servicio con historia conocida de agenesia de sacro entre enero de 1975 y diciembre de 2005. 
En primer lugar se valoró la situación de entrada de todos los pacientes: factores de riesgo (madre diabética), edad a la que recibieron la primera visita urológica, motivo de consulta y estado basal del tracto urinario superior e inferior, malformaciones asociadas y lesión neurológica principal.

Asimismo se ha controlado el tiempo medio de seguimiento, evolución de la continencia clínica y urodinámicamente, seguimiento del tramo urinario superior e inferior con sus complicaciones (atrofia, infecciones, cálculos, reflujo, fístulas) y función eréctil. También se han valorado el número de ingresos hospitalarios por causa urológica de estos pacientes y el número de visitas ambulatorias que han requerido durante el seguimiento.

Todos los pacientes fueron sometidos a estudio urodinámico al menos una vez durante el seguimiento. Para la valoración del tracto urinario superior se realizó ecografía renovesical y/ó renograma isotópico (MAG-3).

Fuera de los parámetros estrictamente urológicos se han registrado otras variables como: hábito intestinal, deambulación, obesidad, estudios realizados y actividad laboral, pareja y descendencia.

\section{RESULTADOS}

En ninguno de los casos se registraron antecedentes de madre diabética. La distribución por sexos fue similar (50\% hombres, $50 \%$ mujeres). Los catorce pacientes presentaban una agenesia total de sacro. La edad media de los pacientes en su primera visita a nuestro servicio fue de 13,2 años (5-33).

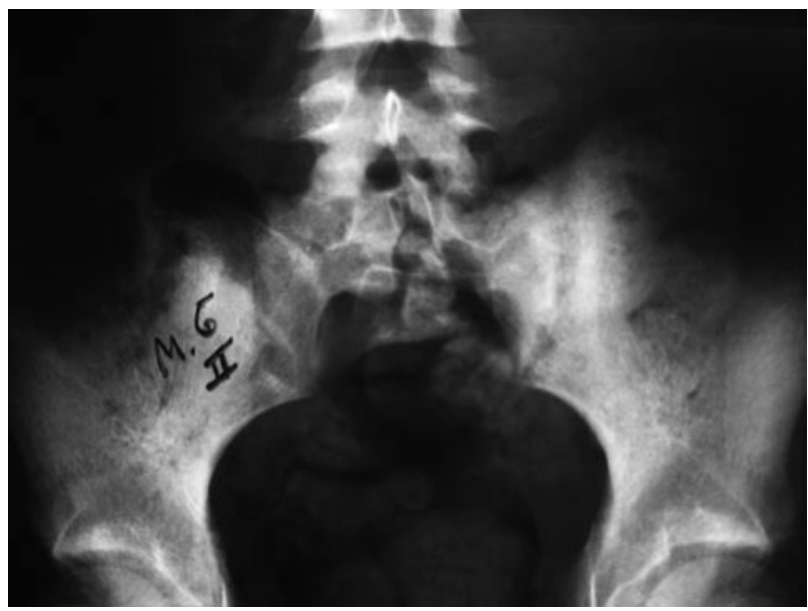

FIGURA 1. Radiografía anteroposterior de pelvis ósea que demuestra ausencia total de sacro.
El motivo de consulta principal fue la incontinencia urinaria $(70 \%)$; el resto de pacientes acudieron por primera vez a nuestras para evaluación y control urológicos (Tabla I).

En el momento de la primera valoración urológica 10 pacientes $(70 \%)$ presentaban ambas unidades renales funcionantes, tres pacientes una ureterohidronefrosis bilateral por reflujo vesicoureteral y un paciente atrofia renal unilateral; asimismo, el $70 \%$ eran incontinentes (cuatro de ellos portadores de sonda vesical y tres se autocateterizaban). Cuatro varones $(60 \%)$ tenían antecedentes de orquidopexia en la infancia por criptorquidia unilateral; además uno tenía hipospadias asociado, otro micropene y otro presentaba aneyaculación.

Once pacientes eran portadores de válvula de derivación ventrículo-peritoneal por hidrocefalia (un paciente presentaba coeficiente intelectual disminuido). El $60 \%$ de los pacientes tenían asociado una disrrafia de la médula espinal (mielomeningocele) por lesión a distintos niveles (la mitad de los casos por defecto entre L3-L5). Un paciente presentaba obesidad mórbida.

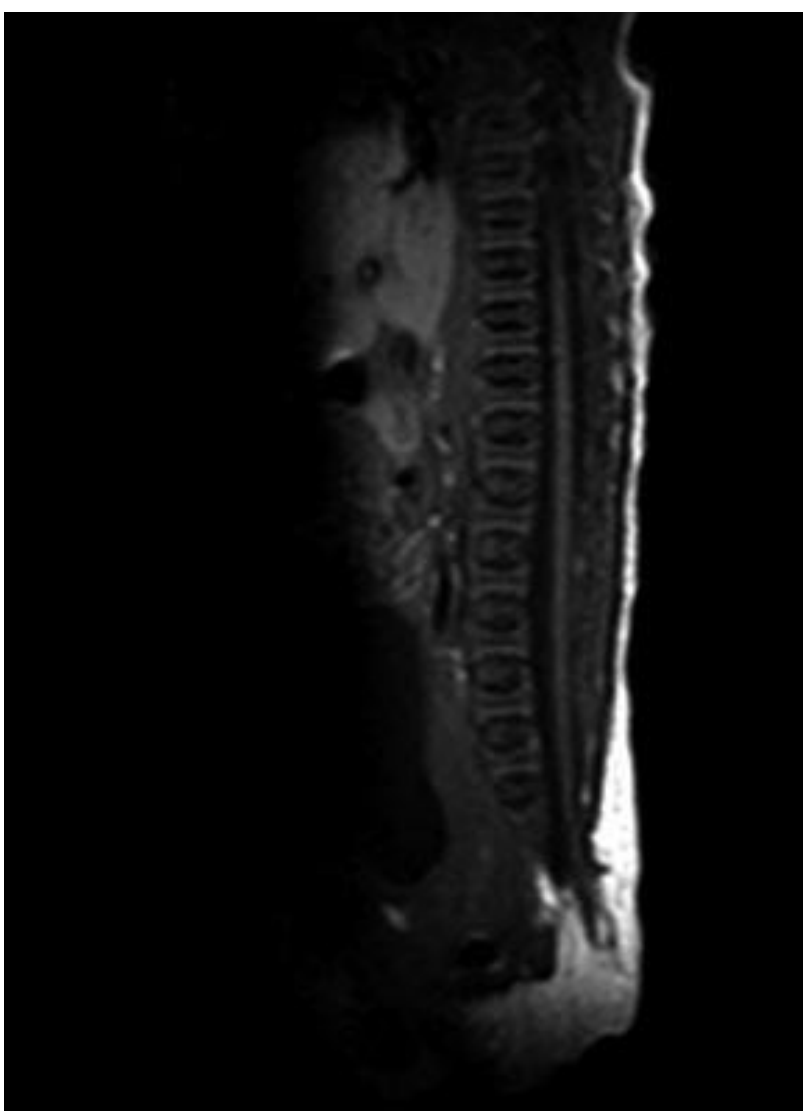

FIGURA 2. Resonancia magnética nuclear que muestra defecto sacro. 
Tras un seguimiento medio de 19,7 años (7-30), el 65\% de los pacientes son continentes 15 mujeres y 4 hombres; todos ellos con tratamiento combinado con anticolinérgicos y cateterismos intermitentes); 3 hombres presentan incontinencia total (dos son portadores de colector urinario y otro de sonda vesical permanente). De las dos mujeres incontinentes una es portadora de sonda vesical (por intolerancia a los autocaterismos) y la otra presenta una incontinencia parcial controlada con anticolinérgicos. El patrón urodinámico más habitual ha sido el de disinergia vesicoesfinteriana (5 casos) con gran residuo postmiccional; dos pacientes presentaban arreflexia detrusoriana (Tabla II).

Durante el tiempo de seguimiento 7 pacientes han mantenido el tracto urinario superior normal, mientras que dos presentaron atrofia renal unilateral progresiva por episodios de pielonefritis de repetición. El $50 \%$ de los pacientes ha presentado en algún episodio de cólico nefrítico a lo largo del seguimiento.

La principal complicación del tracto urinario inferior han sido las infecciones de orina (sintomáticas); todos los pacientes han sufrido algún episodio durante el seguimiento: el germen aislado más frecuentemente ha sido Escherichia coli, seguido de
Pseudomona y Enterococo. Tres pacientes han presentado litiasis vesical subsidiaria de tratamiento quirúrgico. Dos pacientes han experimentado complicaciones por sondajes repetidos (fístula uretro-escrotal, estenosis uretral). De los siete varones del estudio, dos tienen la función eréctil normal.

El motivo urológico más frecuente de ingreso hospitalario ha sido la cirugía programada: 1 cistolitotomía, 2 litotricias endoscópicas, 2 cervicotomías, 2 esfinterotomías. Otras causas de ingreso fueron: sepsis urinaria (2), y hematuria post-traumatismo (1). El número medio de visitas ambulatorias a nuestras consultas durante el seguimiento de estos pacientes ha sido de 1,9 visitas / año.

Asimismo se han controlado otros parámetros fuera del aspecto estrictamente urológico (Tabla III). La deambulación es normal en 6 pacientes, 5 necesitan silla de ruedas y tres ortesis. El hábito intestinal es normal en 5 pacientes, cuatro presentan estreñimiento crónico y 6 incontinencia fecal parcial ó total. Del total del grupo, cuatro pacientes presentan obesidad mórbida. Siete pacientes han realizado estudios de primaria, dos una diplomatura, uno graduado social y uno ingeniería; en la actualidad 3 pacientes son amas de casa, dos trabajan como

TABLA I. DISTRIBUCIÓN DE LAS DISTINTAS VARIABLES AL INICIO DEL SEGUIMIENTO.

\begin{tabular}{|l|l|}
\hline Sexo & $70^{7} / 79$ \\
\hline Tipo agenesia & 14 total / O parcial \\
\hline Madre diabética & 0 \\
\hline Edad media primera visita & 13,2 años (5-33) \\
\hline Motivo consulta & Incontinencia (70\%) \\
& Control Urológico (30\%) \\
\hline Primera valoración urológica: & \\
- Ambas unidades renales funcionantes & $10(70 \%)$ \\
- UHN bilateral por RVU & $3(21 \%)$ \\
- Atrofia renal unilateral & $1(7 \%)$ \\
- Incontinencia & 10 \\
- Criptorquidia & $4(55,5 \%)$ \\
- Otros & Micropene(1), Hipospadias(1), Aneyaculación(1) \\
\hline Lesión neurológica asociada: & $11(79 \%)$ \\
- Válvula ventrículo-peritoneal & $8(60 \%)$ \\
- Mielomeningocele & 1 \\
- Coeficiente intelectual disminuido &
\end{tabular}

UHN: ureterohidronefrosis; RVU: reflujo vesico-ureteral. 
administrativos y uno en la ONCE. Cuatro pacientes tienen pareja estable y 2 pacientes han tenido 2 hijos (parto mediante cesárea en ambos casos). Una pareja está pendiente de adopción.

\section{DISCUSIÓN}

Existen pocos estudios publicados en la literatura urológica sobre el manejo y la evolución de la agenesia sacra en el adulto. La incidencia de esta malformación que presentamos en nuestro trabajo es baja (14 casos / 500000 habitantes) como en otras publicaciones (1-6). Nosotros no hemos encontrado ningún paciente con antecedentes de madre diabética, al contrario que Wilmshurst y cols. (6) que objetivan hasta un $50 \%$ de casos en su serie. También este grupo publica una alta prevalencia de agenesia parcial $(50 \%)$, mientras que en nuestra revisión todos los pacientes presentaban una agenesia total de sacro.

El $60 \%$ de nuestros pacientes presentaban mielomeningocele asociado. Otros grupos publican la triada de Currarino (8) que asocia la agenesia sacra a anomalías anorrectales y masa presacra (teratoma, meningocele). No encontramos ningún caso con esta triple asociación de herencia autosómica dominante.

El principal motivo de consulta fue la incontinencia urinaria. Éste dato coincide con otros autores que demuestran una vejiga neurógena en la mayoría de los pacientes con agenesia sacra (1-7). El principal hallazgo urodinámico en las distintas series es la hiperreflexia del detrusor y la disinergia vesicoesfinteriana. En nuestro estudio el principal patrón urodinámico fue el de disinergia.

La mayoría de nuestros pacientes (70\%) tenían ambos riñones normofuncionantes en el momento de la primera valoración. Llama la atención el deterioro renal que se describe en algunos pacientes incluso en el momento del diagnóstico de la malformación, como Saito y cols. (5) que objetivan un deterioro de la función renal en el $25 \%$ de los casos, incluso un paciente que requirió de hemodiálisis.

\section{TABLA II. TIEMPO DE SEGUIMIENTO Y EVOLUCIÓN CLÍNICA DE LOS PACIENTES.}

\begin{tabular}{|c|c|}
\hline Tiempo medio seguimiento & 19,7 años (7-30) \\
\hline \multicolumn{2}{|l|}{ Continencia urinaria: } \\
\hline - Sí & $9(65 \%): 4_{-} / 5_{-}(\mathrm{Cl}+\mathrm{AC})$ \\
\hline - No & 5 : colector (2), sonda (1_/2_) \\
\hline \multirow[t]{2}{*}{ Urodinámica } & Disinergia vesico-esfinteriana (5) \\
\hline & Arreflexia detrusor (2) \\
\hline \multicolumn{2}{|l|}{ Tracto urinario superior: } \\
\hline - Normal & 7 \\
\hline - Atrofia unilateral & 2 \\
\hline - Cólico nefrítico & 7 (3 Episodios en el mismo paciente) \\
\hline \multicolumn{2}{|l|}{ Tracto urinario inferior: } \\
\hline - Normal & 4 \\
\hline - ITU & 14 \\
\hline - Litiasis vesical & 3 \\
\hline - Otros & Estenosis uretral (1), \\
\hline \multirow[t]{2}{*}{ Función eréctil normal } & Fístula uretro-escrotal (1) \\
\hline & $30 \%$ \\
\hline \multirow[t]{3}{*}{ Causa ingreso hospitalario } & Cirugía programada (7) \\
\hline & Sepsis urinaria (2) \\
\hline & Hematuria post-traumatismo (1) \\
\hline Visitas ambulatorias & 1,9 visitas por paciente / año \\
\hline
\end{tabular}

$\mathrm{Cl}$ : cateterismos intermitentes; $\mathrm{AC}$ : anticolinérgicos. 
TABLA III. EVOLUCIÓN DE LAS VARIABLES NO UROLÓGICAS DEL ESTUDIO.

\begin{tabular}{|l|l|}
\hline \multicolumn{1}{|l|}{} & \\
\hline Deambulación: & \\
\hline - Normal & 6 \\
- Ortesis & 3 \\
- Silla de ruedas & 5 \\
\hline Hábito intestinal: & \\
- Normal & 5 \\
- Estreñimiento & 4 \\
- Incontinencia & 6 \\
\hline Obesidad & 4 \\
\hline Estudios & Primaria (7), Graduado social (1), Diplomatura (2), \\
\hline Actividad laboral & Superior (1).Total: 80\% \\
\hline Pareja & Ama de casa (3), Administrativos (2), ONCE (1). \\
\hline Descendencia & Total: 43\% \\
\hline
\end{tabular}

Tras 20 años de seguimiento medio la mayoría de los pacientes se mantienen continentes $(65 \%)$ y la mitad, tienen conservadas ambas unidades renales. Dado que el deterioro renal es irreversible es importante recalcar el seguimiento continuo que requieren estos pacientes. En este sentido hemos calculado prácticamente dos visitas anuales de media por cada paciente.

Asimismo hemos recogido el número de ingresos hospitalarios que han experimentado nuestros pacientes, siendo en su mayoría por cirugía programada de complicaciones a medio plazo en el seguimiento: litiasis vesical (1 cistolitotomía, 2 litotricias endoscópicas), hipertonía esfinteriana (2 esfinterotomías), obstrucción prostática (2 trigonocervicotomías). Dos ingresos se debieron a sepsis urinaria que requirió de antibioticoterapia endovenosa durante al menos 10 días. Otro paciente presentó un traumatismo renal casual que también necesitó de ingreso para control evolutivo y tratamiento conservador.

\section{CONCLUSIONES}

La agenesia de sacro es una malformación congénita infrecuente que requiere un diagnóstico precoz para evitar complicaciones urológicas a medio plazo. Estos pacientes son subsidiarios de un control urológico de por vida. El principal motivo de consulta es la incontinencia urinaria por vejiga neurógena que puede ser tratada satisfactoriamente en la mayoría de los casos.

\section{BIBLIOGRAFIA y LECTURAS RECOMENDADAS ( ${ }^{*}$ lectura de interés $y^{* *}$ lectura fundamental)}

1. WEIN, A.J.: "Neuromuscular dysfunction of the lower urinary tract and its management. En: Campbell's Urology". Editores: Walsh PC, Retik AB, Vaughan ED, Wein AJ. Ed. Saunders, Philadelphia, 8th edition, pp 931. 2002.

2. BRAREN, V.; JONES, W.B.: "Sacral agenesis: diagnosis, treatment and follow-up of urological complications". J. Urol.; 121:543. 1979

*3. GOTOH, T.; SHINNO, Y.; KOBAYASHI, S. y cols.: "Diagnosis and management of sacral agenesis". Eur. Urol.; 20:287. 1991.

4. GUZMAN, L.; BAUER, S.B.; HALLET, M. y cols.: "Evaluation and management of children with sacral agenesis". Urology; 22:506. 1983.

5. SAITO, M.; KONDO, A.; KATO, K.: "Diagnosis and treatment of neurogenic bladder due to partial sacral agenesis". BJU; 67:472. 1991.

**6. WILMSHURST, J.; KELLY, R.; BORZYSKOWSKI, M.: "Presentation and outcome of sacral agenesis: 20 years of experience". Dev. Med. Child. Neurol.; 41:806. 1999.

7. ORTIZ, M.; ESPEJO, E.; HONRUBIA, B. y cols.: "Incontinencia urinaria como primera manifestación de meningocele sacro anterior gigante". Urod A; 17:103. 2004.

8. LYNCH, S., WANG, Y.; STRACHAN, T. y cols.: "Autosomal dominant sacral agenesis: Currarino syndrome". J. Med. Genet.; 37:561. 2000. 